\title{
Use of human acellular dermal matrix for wound healing in a patient with necrotizing fasciitis, after failure of autologous dermal / epidermal skin graft: A case report
}

\author{
Sergio Mazzei ${ }^{1}$, Alessandro Sindoni ${ }^{2}$, Fausto Famà ${ }^{3}$, Giampietro Bertasi ${ }^{4 *}$, Nimfa J Buizon ${ }^{1}$ and Mohab A Shafei ${ }^{1}$ \\ ${ }^{1}$ Hyperbaric Oxygen Therapy and Wound Care Department, Al Zahra Hospital, Dubai \\ ${ }^{2}$ Department of Public Health and Infectious Diseases, Sapienza University of Rome, Rome, Italy \\ ${ }^{3}$ Department of Human Pathology in Adulthood and Childhood “G. Barresi”, University Hospital of Messina, Messina, Italy \\ ${ }^{4}$ University of Padua, Italy
}

\begin{abstract}
The use of human Acellular Dermal Matrices (hADMs) has been described in patients requiring treatment after skin burns, wounds with bone exposure, complex trauma wounds and breast surgery. We report a clinical case using hADM to close an open wound of the right foot caused by the penetration of part of a shell into the skin followed by necrotizing fasciitis, after multiple unsuccessful treatments using Negative Pressure Wound Therapy, porcine acellular dermal substitutes and a split-thickness skin graft. hADM appeared to be an appropriate adjunct for wound healing process in our patient with chronic open wound failing to heal.
\end{abstract}

\section{Introduction}

Skin and soft-tissue infections (SSTIs) include a variety of pathological conditions which involve the skin and underlying subcutaneous tissues, fascia or muscles, that may also cause necrotizing infections $[1,2]$.

In 1998, the US Food and Drug administration (FDA) classified SSTIs in two main categories: uncomplicated and complicated. Uncomplicated SSTIs are represented by superficial infections as furuncles, abscesses, cellulitis and/or impetigo requiring antibiotic or surgical procedures, whereas complicated SSTIs are represented by deep soft tissue infections such as necrotizing infections, infected ulcers, infected burn and major abscess requiring surgical intervention with drainage and debridement $[3,4]$.

The use of human Acellular Dermal Matrices (hADMs) has been reported mainly after skin burns or cosmetic and reconstructive procedures [5]. These applications can be performed successfully since hADMs have the ability to incorporate into host tissue and allow cellular and vascular ingrowths, without eliciting any biologic inflammatory response and avoiding infection $[6,7]$.

We reported a clinical case using hADM to close an open wound of the right foot caused by necrotizing fasciitis after multiple treatment failures.

\section{Case presentation}

On July 2018, a 48-year-old non-smoker and non-diabetic healthy man had small open wounds of the right leg and plantar foot as a consequence of the penetration of part of a shell into the skin. After 12 hours, he experienced high fever caused by an infection involving locally the skin and subcutaneous tissues up to the muscular fascia (Figure 1). The patient was admitted to the intensive care unit for several surgical procedures (incision, toilette and debridement up to the muscular fascia) of the leg and instep of the right lower limb. After 20 days, the infection seemed to be under control, since the open wound of the leg was closed with good results, but regarding the instep of the foot, the wound failed to close after several different treatments, using Negative Pressure Wound Therapy, porcine acellular dermal substitutes and a split-thickness skin graft. No signs of abnormalities in the vascularity of the foot were noted.

On the 13th of October, the patient was referred for the first time at our Institution for further investigation and treatment. The patient was affected by claustrophobia and denied hyperbaric oxygen therapy. At admission, there was an open wound of the instep of the right foot $(11 \mathrm{~cm} \times 5 \mathrm{~cm}$ in max diameters) with necrotic and slough-macerated tissue in the bed of the wound (Figure 2). The cultural swab showed Carbapenem-resistant Pseudomonas Aeruginosa contamination; in accordance with the cultural swab, the patient was treated with Cyprofloxacin $500 \mathrm{mg}$ twice a day for two weeks. After fifteen days of debridement using curette and super-oxized solution as bridging treatment in outpatient service, the patient underwent surgical procedure on the 30th of October 2018 using hADM (Figure 3).

The patient underwent periodic changes of the paraffin gauze dressing used to cover the dermal substitute. After 50 days from the surgery, we decided to have follow-up only once a week for one month and once every 2 weeks for the consecutive 2 months until the end of

*Correspondence to: Giampietro Bertasi, Via Molino 43, San Pietro in Cariano, Italy, Tel: +393456001037; E-mail: bertasi.g@sis.it

Key words: human Acellular Dermal Matrices, wound healing, necrotizing fasciitis

Received: July 04, 2019; Accepted: July 15, 2019; Published: July 19, 2019 
Mazzei S (2019) Use of human acellular dermal matrix for wound healing in a patient with necrotizing fasciitis, after failure of autologous dermal / epidermal skin graft: A case report

February 2019, when the patient has been discharged (Figure 3). At the end of the treatment the wound was replaced by elastic tissue with no scar or other signs of recurrence (Figures 4 ).

\section{Discussion}

Re-cellularization process is complex, since it represents a balance between cellular migration and healing, avoiding a destructive action [8]. Since 2012, hADM grafts have been proposed for treatment of skin burn injuries, as an alternative for autograft skin and cryopreserved skin, because of their potential to be biocompatible scaffold for new tissue growth: in particular, Chen et al. [9] described its use for treating

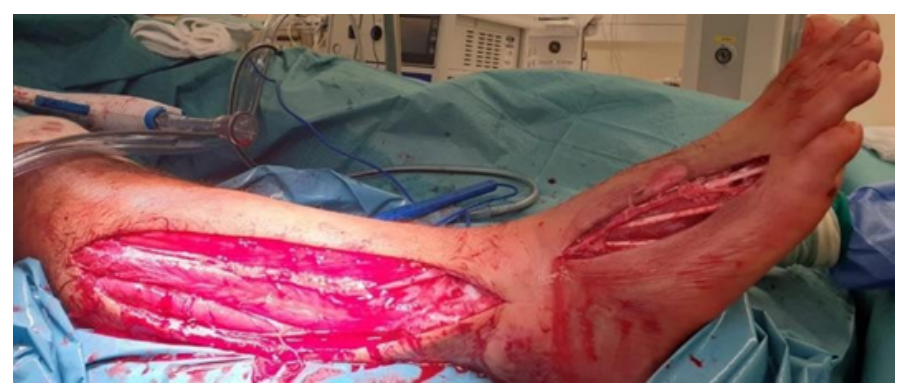

Figure 1. Infection involving locally the skin and subcutaneous tissues up to the muscular fascia

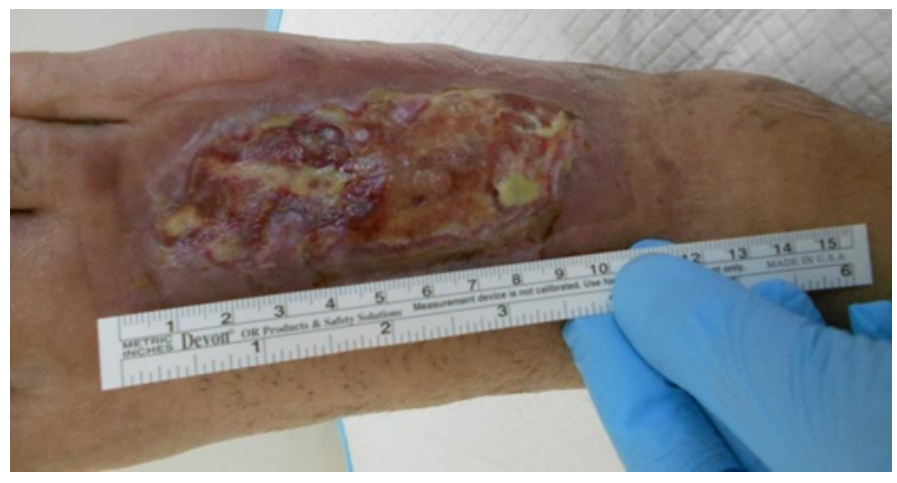

Figure 2. Open wound of the instep of the right foot

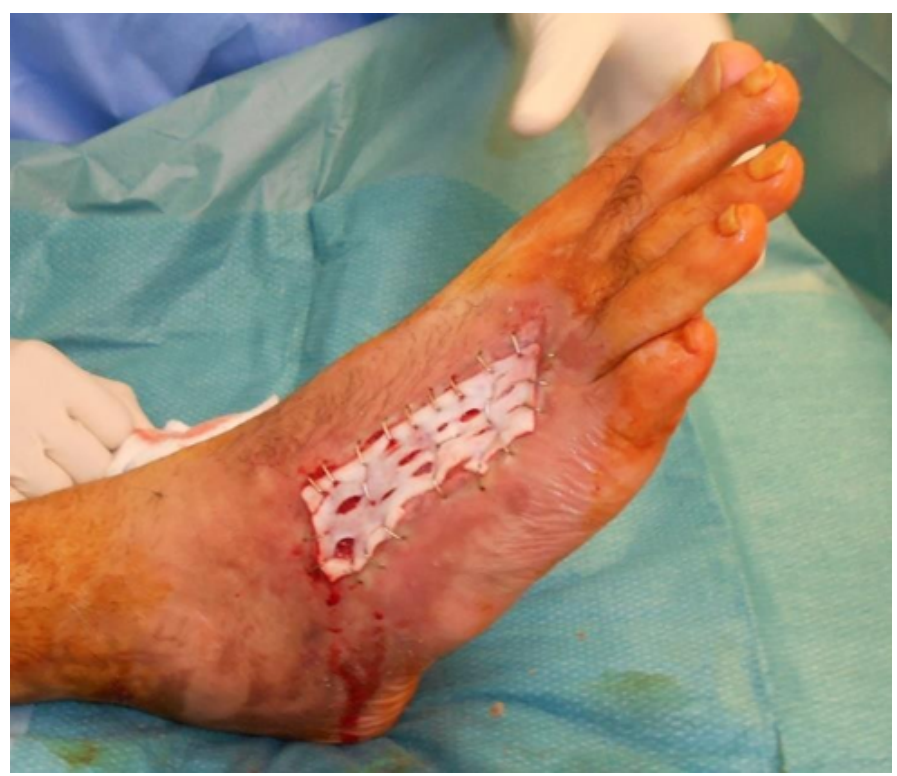

Figure 3. Patient underwent surgical procedure

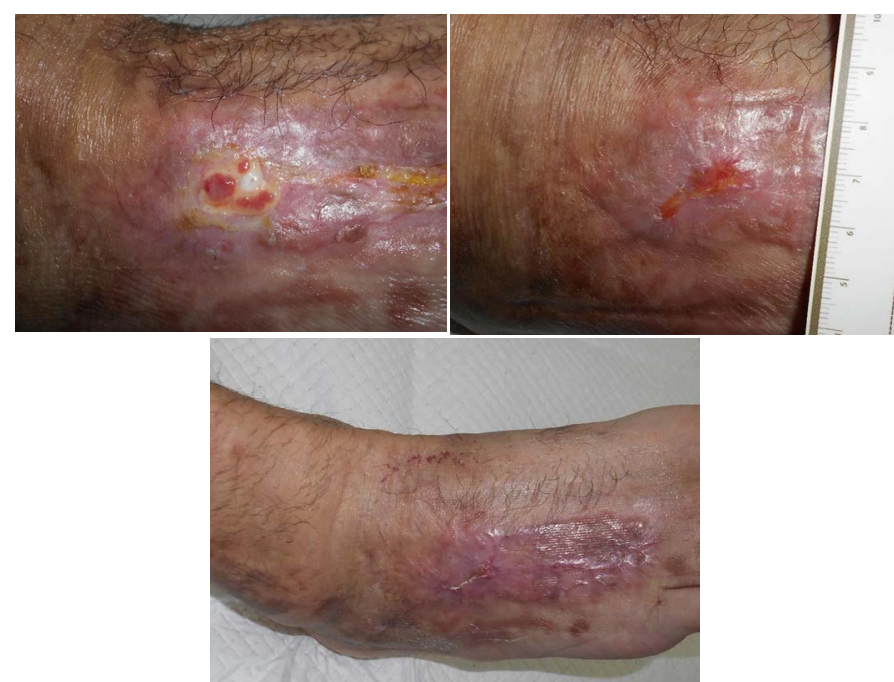

Figure 4. At the end of the treatment the wound was replaced by elastic tissue with no scar or other signs of recurrence

a scar resulting from second- and third-degree burns in a 33-year-old female patient. Cole WE reported a 56-year-old diabetic woman with a Wagner grade 3 ulcer of the right heel treated with hADM: her past history was relevant for ulceration with moderate drainage of the right limb and ulceration-osteomyelitis in the contralateral limb. Rigorous wound care, including hospitalization, surgical incision and drainage, intravenous antibiotic drug therapy, vacuum-assisted therapy, and hADM graft were used to heal the wound, save her limb, and restore her activities of daily living [10]. Contemporary to this report, Walters et al conducted a 16-week, multicentre, randomized, controlled trial to evaluate the healed ulcer rate of a hADM (DermACELL) compared with conventional care and a second hADM (Graftjacket) in the treatment of full-thickness diabetic foot ulcers. At 16 weeks, the DermACELL arm had a significantly higher proportion of completely healed ulcers than the conventional care, but the differences with the Graftjacket arm (67.9\% vs $47.8 \%)$ did not reach the statistical significance. Interestingly, the DermACELL arm exhibited in a trend wise manner a greater average percent reduction in wound area than the conventional care arm and the Graftjacket arm. No differences regarding adverse events were noted between the groups. These results suggested the advantage to use DermACELL ADM in the treatment of diabetic foot ulcers [11]. Other settings reported in literature include wound with bone exposure [12], complex trauma wound [13] and breast surgery [14].

The success reported with hADM may be due to the intrinsic characteristics and sterility of the material employed. The procedure to prepare the hADM requires the use of a solution of non - denaturing anionic detergent (as N-Laurolsarcosinate), recombinant and antibiotics, resulting in a material that exhibits at least $97 \%$ nucleic acid removal, while maintaining biomechanical strength. hADM grafts are included in glycerol, stored at room temperature, and they need no rehydration $[9,15]$. The peculiarity of our case report is the use of hADM in a patient with open wound of the right foot complicated by necrotizing fasciitis, after multiple treatment failures using Negative Pressure Wound Therapy, pork acellular dermal substitutes (xenograft) and a split-thickness skin graft. hADM appears to be an appropriate adjunct for wound healing process in our patient with chronic open wound failing to heal. 
Mazzei S (2019) Use of human acellular dermal matrix for wound healing in a patient with necrotizing fasciitis, after failure of autologous dermal / epidermal skin graft: A case report

\section{References}

1. May AK (2009) Skin and soft tissue infections. Surg Clin North Am 89: 403-420, viii. [Crossref]

2. Ustin JS1, Malangoni MA (2011) Necrotizing soft-tissue infections. Crit Care Med 39: 2156-2162. [Crossref]

3. Merlino JI, Malagoni MA (2007) Complicated skin and soft-tissue infection: diagnostic approach and empiric treatment options. Clev Clin J Med 74: S21-S28. [Crossref]

4. Napolitano LM1 (2009) Severe soft tissue infections. Infect Dis Clin North Am 23: 571-591. [Crossref]

5. Bertasi G, Cole W, Samsell B, Qin X, Moore M (2017) Biological incorporation if human acellular dermal matrix used in Achilles tendon repair. Cell Tissue Bank 18: 403- 411. [Crossref]

6. Wainwright DJ (1995) Use of an acellular allograft dermal matrix (AlloDerm) in the management of full-thickness burns. Burns 21: 243-248. [Crossref]

7. Lattari V, Jones LM, Varcelotti JR, Latenser BA, Sherman HF, et al. (1997) The use of a permanent dermal allograft in full-thickness burns of the hand and foot: a report of three cases. J Burn Care Rehabil 18: 147-155. [Crossref]

8. Orenstein S, Qiao Y, Kaur M, Klueh U, Kreutzer D, et al. (2010) In vitro activation of human peripheral blood mononuclear cells induced by human biologic meshes. J Surg Res 158: 10-14. [Crossref]
9. Chen SG, Tzeng YS, Wang CH (2012) Treatment of severe burn with DermACELL(®), an acellular dermal matrix. Int J Burns Trauma 2: 105-109. [Crossref]

10. Cole WE (2016) DermACELL: Human Acellular Dermal Matrix Allograft A Case Report. J Am Podiatr Med Assoc 106: 133-137. [Crossref]

11. Walters J, Cazzell S, Pham H, Vayser D, Reyzelman A (2016) Healing Rates in Multicentre Assessment of a Sterile, Room Temperature, Acellular Dermal Matrix Versus Conventional Care Wound Management and an Active Comparator in the treatment of Full-Thickness Diabetic Foot Ulcers. Eplasty16: e10. [Crossref]

12. Roussalis JL (2014) Novel use of an acellular dermal matrix allograft to treat a chronic scalp wound with bone exposure: a case study. Int J Burns Trauma 4: 49-52. [Crossref]

13. Shitrit SB, Ramon Y, Bertasi G (2014) Use of a novel acellular dermal matrix allograft to treat complex trauma wound: a case study. Int J Burns Trauma 4: 6265. [Crossref]

14. Cheng A, Saint-Cyr M (2012) Comparison of different ADM materials in breast surgery. Clin Plast Surg 39: 167-175. [Crossref]

15. Bullocks JM (2014) DermACELL: a novel and biocompatible acellular dermal matrix in tissue expander and implant-based breast reconstruction. Eur J Plast Surg 37: 529538. [Crossref]

Copyright: (C2019 Mazzei S. This is an open-access article distributed under the terms of the Creative Commons Attribution License, which permits unrestricted use, distribution, and reproduction in any medium, provided the original author and source are credited. 